\title{
Optimal surplus and minimum benefits for a defined contribution pension plan: a mean-variance approach
}

\author{
Charles I. Nkeki \\ Department of Mathematics, Faculty of Physical Sciences, University of Benin, Nigeria
}

\begin{abstract}
In this paper, we study a mean-variance portfolio selection problem, optimal surplus, Minimum Pension Benefits (MPB) and consumption plan of a defined contribution pension scheme. The problem is formulated as a tri-objective stochastic problem of mean-variance techniques. The problem is solved using dynamic programming approach. The aim of the fund manager is to maximize Pension Plan Member's (PPM) expected MPB and expected surplus, and at the same time minimize the consumption and portfolio risks. We find the efficient frontier to be nonlinear and parabolic in shape. We further show that the optimal portfolio depend linearly on consumption plan and linearly on MPB. The aggregate optimal pension benefits accrued to a plan member at retirement and life-time consumption of the plan member are obtained. Some numerical illustration of our models were obtained.
\end{abstract}

Keywords Mean-Variance, Stochastic Funding, Minimum Pension Benefits, Aggregate Pension Benefits, Tri-objective

AMS 2010 subject classifications 91B28, 91B30, 91B70, 93E20

DOI: $10.19139 /$ soic.v6i4.367

\section{Introduction}

Pension fund management is relatively a long period of time. So, it should be strategically plan to ensure high returns for plan members. It is imperative for plan members to know at the point of entrance into the plan, the expected minimum benefits that will accrued to him or her at retirement. It is also vital for a PPM to know the level of risks associated with the investment portfolio.

In this paper, we consider a mean-variance portfolio selection problem, optimal surplus, Minimum Pension Benefits (MPB) and optimal consumption plans for a defined contribution pension scheme. The surplus arising from the investment will be shared between the PPM and the fund manager in accordance with the agreed rate. The aim of the fund manager is to maximize surplus and the MPB at the same time minimize the consumption and portfolio risks.

In the related literature, [15], considered a Defined Benefit (DB) plans and modeled it as a linear-quadratic optimal control problems. [26] studied a mean-variance optimization model. The techniques was used to compare securities and portfolios based on a tradeoff between their expected return and its variance. [8] and [19] considered a mean-variance portfolio problem in pension plans from a static point of view. [7] studied a dynamic case of the model for asset and liability management under the mean-variance criteria. [23] studied the benefits of the DB plan by assuming that the benefits are stochastic. They also assumed that benefit is a non-tradeable asset. The existence of correlation between the sources of uncertainty in the benefits and in the asset returns are also considered in their work.

\footnotetext{
${ }^{*}$ Correspondence to: Charles I. Nkeki (Email: nkekicharles2003@gmail.com). Department of Mathematics, Faculty of Physical Sciences, University of Benin, P. M. B. 1154, Benin City, Edo State, Nigeria.
}

ISSN 2310-5070 (online) ISSN 2311-004X (print)

Copyright (C) 2018 International Academic Press 
It has been a challenge in the literature on how to translate the mean-variance approach from the static state to the dynamic state. Merton tried to translate the problem to dynamic setting but does not exactly fit the structure of a mean-variance approach. The problem of determining the minimum variance on trading strategy in continuoustime framework has been studied by [36] via the Martingale approach. [1] used the same approach in a more general framework. The papers by [38] and [25] successfully carried out the translation from static state to dynamic setting both in continuous time and discrete time. $\mathrm{Li}$ and $\mathrm{Ng}$ (2000) solved a mean-variance optimization problem in a discrete-time multi-period framework. [38] considered a mean-variance in a continuous-time framework. They shown the possibility of transforming the difficult problem of a mean-variance optimization problem into a tractable one, by embedding the original problem into a stochastic linear-quadratic control problem, that can be solved using standard methods. These approaches have been extended and used by many in the financial literature, see for instance, [3], [7], [18], [23], [37]. [23] follow the formulation of [38] but with some modifications to study a DB pension plan with the inclusion of the supplementary cost as a control variable in addition to the presence of several risky assets. In this paper, we follow the formulation of [38] and [23] but with some modifications toward studying a DC pension plan with the inclusion of investment strategy, the MPB and consumption rate as control variables. Although, [23] supplementary cost and our consumption rate have the same features but in different setting. In our own setting, consumption include management cost and withdrawal on the part of the PPM. We adopt the Hamilton-Jacobi-Bellmam principles in the optimization process. [29] considered a mean-variance portfolio selection problem with inflation hedging strategy for a defined contributory pension scheme. In the paper, the optimal wealth which involves a cash account and two risky assets for the pension plan member was established. It was found that inflation-linked bond is a suitable asset for hedging inflation risks in an investment portfolio. [?] examines a mean-variance portfolio selection problem with fixed salary or income and inflation protection strategy in the accumulation phase of a defined contribution pension plan. For more on a mean-variance portfolio selection problem, see [30], [31] and [33].

This paper aims at studying optimal surplus, MPB, optimal total benefit and optimal consumption plan under the context of a defined contribution pension plan with stochastic funding in a DC pension scheme under meanvariance efficient approach. [18] and [37] assumed a constant flow of contributions into the pension scheme which will not be applicable to a time-dependent salary earners in pension scheme. We assume that the contribution of the PPM grows as the salary grows over time. In this paper, the contributions of the plan member is assumed to be stochastic.

There are extensive literature that exist on the area of accumulation phase of DC pension plan and optimal investment strategies. This can be found in [28], [10], [5], [24], [17], [11], [2], [6], [13], [4], [12], [37], [29], [31]. For optimal pension management of a DC pension plan, see [34] and [35].

The remainder of this paper is organized as follows. In section 2, we present financial market models, the salary process and the surplus process. In section 3 , we presents the aggregate pension benefits. In section 4, we present the mean-variance formulation of our problem. Section 5 presents the optimization problem. In section 6 , we present the optimal feedback controls. Section 7 presents the efficient frontier of the optimal terminal expected surplus. In section 8 , we presents aggregate optimal final pension benefits and optimal final consumption plan of a PPM at retirement. Section 9 presents the numerical examples of our models. Finally, section 10 concludes the paper.

\section{The Models}

In this section, we present the financial market models, the salary and wealth process, and the dynamics of the surplus plans.

\subsection{Financial market models}

Let $(\Omega, \mathcal{F}, \mathbf{P})$ a probability space and $\bar{W}(t)=\left(W^{B}(t), W^{S}(t), W^{Y}(t)\right)^{\prime}=\left(W(t), W^{Y}(t)\right)^{\prime}$ defined on a given filtered probability space $(\Omega, \mathcal{F}, \mathbf{F}(\mathcal{F}), \mathbf{P})$, where $W(t)=\left(W^{B}(t), W^{S}(t)\right), \mathcal{F}_{t}=\sigma\left(W(s), W^{Y}(s): s \leq t\right)$ and $\mathbf{F}(\mathcal{F})=\left\{\mathcal{F}_{t}: t \in[0, T]\right\}$, is a 3-dimensional Brownian motion with respect to bond risk, stock market risk and 
salary risk, respectively at time, $t$. $\mathbf{P}$ denotes the real world probability measure, $T$ the retirement time and the sign ', denotes transpose.

The fund manager manage the stochastic fund contributed by the PPMs in the planning interval $[0, T]$, by means of a portfolio characterized by

$$
\begin{aligned}
S_{0}(t) & =\text { price process of cash account at time } t, \\
Z(t) & =\text { price process of bond at time } t, \text { and } \\
S(t) & =\text { price process of stock at time } t .
\end{aligned}
$$

2.1.1. Cash account, bond and stock dynamics The dynamics for cash account, bond and stock are given respectively by

$$
\begin{gathered}
d S_{0}(t)=r S_{0}(t) d t, S_{0}(0)=1, \\
d Z(t)=Z(t)\left(\mu_{Z} d t+\sigma_{Z} d W(t)\right), Z(0)=z_{0} \in \mathbb{R}_{+},
\end{gathered}
$$

where

$$
\left\{\begin{array}{l}
\mu_{Z}=r-\alpha T+\frac{1}{2}(\nu T)^{2} \\
\sigma_{B}=-\nu T
\end{array}\right.
$$

where $r$ is the short interest rate, $\alpha$ the drift of the forward interest rate, $\nu$ the volatility of the forward interest rate and $\sigma_{Z}=\left(\sigma_{B}, 0\right)$.

$$
d S(t)=S(t)(\mu d t+\sigma d W(t)), S(0)=s_{0} \in \mathbb{R}_{+},
$$

where $\sigma=\left(\sigma_{S} \rho, \sigma_{S} \sqrt{1-\rho^{2}}\right), \mu>0$ rate of return of the stock, $\sigma_{S}$ the volatility of stock, $\rho \in(-1,1)$ correlation coefficient of source of risks from bond, $W^{B}(t)$ and stock, $W^{S}(t)$.

Then, the volatility matrix

$$
\Sigma:=\left(\begin{array}{cc}
\sigma_{B} & 0 \\
\rho \sigma_{S} & \sqrt{1-\rho^{2}} \sigma_{S}
\end{array}\right)
$$

corresponding to the two risky assets, satisfies $\operatorname{det}(\Sigma)=\sigma_{S} \sigma_{B} \sqrt{1-\rho^{2}} \neq 0$.

Therefore, the market is complete and there exists a unique market price of risks, $\theta$ satisfying

$$
\theta:=\left(\begin{array}{c}
\theta_{B} \\
\theta_{S}
\end{array}\right)=\left(\begin{array}{c}
\theta_{B} \\
\frac{\mu-r-\theta_{B} \rho \sigma_{S}}{\sigma_{S} \sqrt{\left(1-\rho^{2}\right)}}
\end{array}\right),
$$

where $\theta_{B}$ is the bond price of risks and $\theta_{S}$ the market price of stock risks.

2.1.2. The stochastic salary process Let $Y(t)$ be the salary process of a PPM at time $t$, then the dynamics of $Y(t)$ is driven by the following stochastic differential equation:

$$
d Y(t)=Y(t)\left(\beta d t+\bar{\sigma}_{Y} d \bar{W}(t)\right), Y(0)=y_{0} \in \mathbb{R}_{+},
$$

where $\beta>0$ is the expected growth rate of the salary, $\bar{\sigma}_{Y}$ which is equal to $\left(\sigma_{Y_{1}}, \sigma_{Y_{2}}, \sigma_{Y_{3}}\right)=\left(\sigma_{Y}, \sigma_{Y_{3}}\right), \sigma_{Y}=$ $\left(\sigma_{Y_{1}}, \sigma_{Y_{2}}\right), \sigma_{Y_{1}}$ volatility of the salary of a PPM arising from the uncertainty of bond, $W^{B}(t), \sigma_{Y_{2}}$ volatility of the salary of a PPM arising from the uncertainty of stock market, $W^{S}(t), \sigma_{Y_{3}}$ the volatility of the salary process arising from the source of salary risk, $W^{Y}(t)$ at time $t$.

Suppose the proportion $c>0$ of the salary process is a contribution of the PPM into the scheme, then $c Y(t)$ is the amount of fund contributed into the scheme by a PPM at time $t$.

\subsection{The wealth and surplus process}

Let $\Phi(t)$ be the investment process at time $t, \tilde{C}(t)$ the consumption process at time $t$ (i.e., withdrawal process from the pension scheme by the PPM) and $B(t)$ the MPB at time $t$. The amount of fund invested in stock, $S(t)$ at time, $t$ is denoted by $\Delta_{S}(t)$ and fund invested in bond is $\Delta_{B}(t)$. The remainder, $\Phi(t)-\Delta_{B}(t)-\Delta_{S}(t)$ is invested in cash account at time $t$. 
2.2.1. The wealth process Before expressing the dynamics of the wealth process, we first give the following definition.

\section{Definition 1}

Let $\mathcal{C}$ be the set of admissible strategies and $\mathcal{C}_{\Delta}, \mathcal{C}_{c}$ and $\mathcal{C}_{M P B}$ with respect to investment, consumption and MPB, respectively be subsets of $\mathcal{C}$. We say:

(i) $\Delta \in \mathcal{C}_{\Delta}$ if

$$
E \int_{0}^{T} \Delta(t) \Delta(t)^{\prime} d t<\infty
$$

where $E$ is the expectation operator and $\Delta(t)=\left(\Delta_{B}(t), \Delta_{S}(t)\right)$ is a control process adapted to filtration $\left\{\mathcal{F}_{t}\right\}_{t \geq 0}$, $\mathcal{F}_{t}$-measurable.

(ii) $B \in \mathcal{C}_{M P B}$ if

$$
E \int_{0}^{T} B(t)^{2} d t<\infty
$$

For the rate of consumption $C(t)$, we assume that is a non-negative and bounded above with upper bound being $\Theta$ such that $0 \leq C(t) \leq \Theta<\infty$.

A strategy $u(\cdot)=\{\Delta(t), B(t), C(t): t \geq 0\}$ which is progressively measurable with respect to $\{\bar{W}(t), P(t)$ : $0 \leq s \leq t\}$ is referred to as admissible strategy. We denote the collection of all admissible strategies by $\mathcal{A}$. It then follows that the set of all admissible strategies $\mathcal{A}$ can be defined as follows:

$$
\begin{aligned}
& \mathcal{A}:=\left\{u(t)=(\Delta(t), B(t), C(t)) \in \mathcal{R}^{2} \times \mathcal{R} \times \mathcal{R}:\right. \\
& \left.E \int_{0}^{T} \Delta(t) \Delta(t)^{\prime} d t<\infty ; E \int_{0}^{T} B(t)^{2} d t<\infty ; 0 \leq C(t) \leq \Theta<\infty\right\} .
\end{aligned}
$$

2.2.2. Investment and wealth processes The investment process $\Phi(t)$ at time $t$ is given by the following dynamics

$$
d \Phi(t)=(r \Phi(t)+\Delta(t) \lambda) d t+\Delta(t) \Sigma d W(t), \Phi(0)=\Phi_{0} \in \mathbb{R}_{+}
$$

where $\lambda=\left(\sigma_{1} \theta_{B}, \mu-r\right)^{\prime}$.

Let $\Psi(t)$ be the wealth process at time $t$. Since a PPM make flow of contributions $c Y(t)$ into the scheme at time $t$ and assume that withdrawals $\tilde{C}(t)$ (which we referred to here as consumption) are made from the wealth generated at time $t$, then we have the following:

$$
\Psi(t)=\Phi(t)+c Y(t)-\tilde{C}(t)
$$

where $\tilde{C}(t)=\int_{0}^{t} \tilde{C}(s) d s$. Finding the differential of both sides of (11) and using (6), (10), we have the wealth dynamics as follows:

$$
\begin{aligned}
& d \Psi(t)=(r \Phi(t)+\Delta(t) \lambda+c \tilde{\beta} Y(t)-\tilde{C}(t)) d t+\left(c \sigma_{Y}^{\prime} Y(t)+\Sigma^{\prime} \Delta(t)^{\prime}\right)^{\prime} d W(t) \\
& +c \sigma_{Y_{3}} Y(t) d W^{Y}(t), \Psi(0)=\Psi_{0} \in \mathbb{R}_{+} .
\end{aligned}
$$

Now, with this wealth dynamics, we determined the surplus process of the stakeholders and is presented in the following subsection.

2.2.3. The surplus process Let $X(t)$ be the surplus process of a PPM at time $t$. Then,

$$
X(t)=\Psi(t)-B(t),
$$

where $B(t)=\int_{0}^{t} B(s) d s$ 
Finding the differential of both sides of (13), we have the dynamics of the surplus process to be

$$
d X(t)=d \Psi(t)-d B(t) .
$$

Substituting (12) into (14), we have

$$
\begin{aligned}
& d X(t)=(r \Phi(t)+\Delta(t) \lambda+c \tilde{\beta} Y(t)-\tilde{C}(t)-B(t)) d t+\left(c \sigma_{Y}^{\prime} Y(t)+\Sigma^{\prime} \Delta(t)^{\prime}\right)^{\prime} d W(t) \\
& +c \sigma_{Y_{3}} Y(t) d W^{Y}(t), X(0)=x_{0} \in \mathbb{R}_{+},
\end{aligned}
$$

(15) can be re-written by using the fact that $\Phi(t)=X(t)+B(t)-c Y(t)+\tilde{C}(t)$ to obtain the following:

$$
\begin{aligned}
& d X(t)=(r X(t)+\Delta(t) \lambda+c \beta Y(t)-C(t)+(r-1) B(t)) d t \\
& +\left(c \sigma_{Y}^{\prime} Y(t)+\Sigma^{\prime} \Delta(t)^{\prime}\right)^{\prime} d W(t)+c \sigma_{Y_{3}} Y(t) d W^{Y}(t), X(0)=x_{0} \in \mathbb{R}_{+},
\end{aligned}
$$

where $C(t)=(1-r) \tilde{C}(t)$ and $\beta=(\tilde{\beta}-r)$.

\section{Remark 1}

From now on we shall be referring to $C(t)$ as consumption plan and having it in mind that $C(t)=(1-r) \tilde{C}(t)$.

Next, we determine the aggregate pension benefit that will accrued to a PPM at time $t$. The aggregate pension benefit measure the sum of the MPB and fraction of surplus to be receive by PPM at time $t$.

\section{Total Pension Benefits}

Let $F(t)$ be the total pension benefits of the PPM at time, $t \in[0, T]$ and $h$ the fixed fraction of the surplus that will be received by the fund manager. At time $T$, the fund manager will make two payment to the PPM. The first payment of MPB, $B(t)$. The second payment is a fixed fraction of the surplus $\Theta(t, X)=(1-h) X(t)$ (the fraction of the difference between the terminal wealth $\Phi(t)$ of the managed portfolio and the MPB, $B(t))$. Therefore, the total benefit to a PPM at time $T$ is

$$
F(T)=B(T)+(1-h) X(T)=B(T)+\Theta(T, X),
$$

where $\Theta(T, X)=(1-h) X(T)$ is the surplus at the final time, $T$.

\section{Remark 2}

For $h=0$, it implies that the fund manager does not keep any profit from the surplus, so introduction of the MPB is more an obstacle for the fund manager, since MPB may induce a significant utility loss for quadratic risk tolerant investors (see [20] for relative risk averse investor). On the other hand, if $h=1$, it implies that the PPM will receive only the MPB, no matter the final surplus, which is unreasonable. In order to avoid these trivial cases, we therefore assume that $h \in(0,1)$.

The flow of total pension benefits, $F(t)$ is defined by

$$
F(t)=\left\{\begin{array}{l}
B(t), \text { if } 0 \leq t \leq T_{0}<T, \\
B(t)+\Theta\left(t,\left.X(t)\right|_{t-T, t}\right), \text { if } t \geq T,
\end{array}\right.
$$

where $T_{0}$ is the time of voluntary retirement.

\section{The Mean-Variance Formulation}

The objectives of the fund manager in this paper, is triple. First, to maximize the value of expected final surplus, $E(X(T))$ and to minimize expected discounted consumption process. Secondly, to maximize the MPB at the terminal time, $T$. Thirdly, to minimize the variance of the final surplus, $\operatorname{Var}(X(T))$. This involves a multi-objective 
optimization problem with three criteria, $J_{1}, J_{2}$ and $J_{3}$ (for two criteria, see [23]):

$$
\begin{aligned}
& \min _{(\Delta, B, C) \in \mathcal{G}_{x_{0}, B_{0}, C_{0}}}\left(J_{1}(\Delta, C, B), J_{2}(\Delta, C, B), J_{3}(\Delta, C, B)\right)= \\
& \min _{(\Delta, B, C) \in \mathcal{G}_{x_{0}, B_{0}, C_{0}}}\left(-E(X(T)),-\int_{0}^{T} B(t)^{2} d t, E \int_{0}^{T} e^{-\eta t} C^{2}(t) d t+\operatorname{Var}(X(T))\right)
\end{aligned}
$$

subject to (16). Here $\eta \in \mathbb{R}_{+}$denotes the discount preference rate of consumption, $\mathcal{G}_{x_{0}, B_{0}, C_{0}}$ is the set of measurable processes $(\Delta, B, C)$, where $\Delta$ satisfies (7), $C$ satisfies (9) and $B$ satisfies (8) such that (16) admit a unique solution, $\mathcal{F}_{t}-$ measurable, and adapted to the filtration $\left\{\mathcal{F}_{t}\right\}_{t \geq 0}$. Note that problems (16) and (18) are mean variance (MV) problem similar to the one studied in [23] and [38], but with additional control variables, MPB, $B(t)$ and consumption plan process, $C(t)$. Let $\Delta^{*}$ be an efficient portfolio, $C^{*}$ an efficient consumption rate and $B^{*}$ an efficient MPB. The admissible control process $\left(\Delta^{*}, B^{*}, C^{*}\right)$ is Pareto efficient if there does not exist admissible process $(\Delta, B, C)$ such that $J_{1}(\Delta, C, B) \leq J_{1}\left(\Delta^{*}, B^{*}, C^{*}\right)$,

$J_{2}(\Delta, C, B) \leq J_{2}\left(\Delta^{*}, B^{*}, C^{*}\right), J_{3}(\Delta, C, B) \leq J_{3}\left(\Delta^{*}, B^{*}, C^{*}\right)$ such that at least one of the inequalities hold strictly. The pairs $\left(J_{1}\left(\Delta^{*}, B^{*}, C^{*}\right), J_{2}\left(\Delta^{*}, B^{*}, C^{*}\right)\right.$,

$\left.J_{3}\left(\Delta^{*}, B^{*}, C^{*}\right)\right) \in \mathbb{R}^{3}$ form the Pareto frontier. [9] stipulated that a situation whereby the objective functionals defining the multi-objective optimization are convex, the Pareto efficient points can be obtained by solving a scalar efficient control problem provided the dynamics remain unchanged and the objective functional is a convex combination of the original cost functionals, (see [23]). In this paper, the Pareto points and controls in (16) are linear, this implies that $J_{1}, J_{2}$ and $J_{3}$ are indeed convex. Hence, problems (16) and (18) are equivalent to the scalar problems

$$
\begin{aligned}
& \min _{(\Delta, B, C) \in \mathcal{G}_{x_{0}, B_{0}, C_{0}}} J_{1}(\Delta, C, B)+J_{2}(\Delta, C, B)+\xi J_{3}(\Delta, C, B)= \\
& \min _{(\Delta, B, C) \in \mathcal{G}_{x_{0}, B_{0}, C_{0}}-\left(E(X(T))+\int_{0}^{T} B(t)^{2} d t\right)} \\
& +\xi\left(E \int_{0}^{T} e^{-\eta t} C^{2}(t) d t+\operatorname{Var}(X(T))\right),
\end{aligned}
$$

subject to (16), where $\xi \in \mathbb{R}_{+}$is a weight parameter. As $\xi$ varies in the interval $(0, \infty)$, the solutions of (19) admits the Pareto frontier. We observe that $\xi$ guides the fund manager on how to transfer linear units of risk to units of expected surplus, and vice versa, see [23]. The size of $\xi$ shows which one of the objectives is of paramount interest to the fund manager, to reduce risk or to increase surplus and MPB.

Problem (19) is not a standard stochastic optimal control problem as a result of the presence of the quadratic form $E(X(T))^{2}$ in the variance term. In this case, the dynamic programming approach cannot be applied. Following [38], [25] and [23], we have the following stochastic-linear-quadratic problem

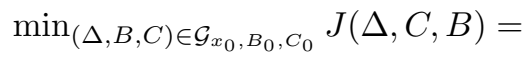

$$
\begin{aligned}
& \left.\min _{(\Delta, B, C) \in \mathcal{G}_{x_{0}, B_{0}, C_{0}}} E\left(X^{2}(T)\right)-2 \varphi X(T)\right)+E \int_{0}^{T}\left(e^{-\eta t} C^{2}(t)-B^{2}(t)\right) d t,
\end{aligned}
$$

subject to (16), where $\varphi \in \mathbb{R}$.

\section{Theorem 1}

Let $\xi \in \mathbb{R}_{+}$and $\left(\Delta^{*}, B^{*}, C^{*}\right)$ an optimal control of (16) and (20) with associated optimal surplus $X^{*}$. Then $\left(\Delta^{*}, B^{*}, C^{*}\right)$ is also an optimal control of (16) and (19), if $\varphi=\frac{1}{2 \xi}+E\left(X^{*}(T)\right)$.

Proof: Omitted since is similar to that provided by [23].

Theorem 1 tells us that optimal solution of problem (16) and (19) can be found by solving problem (16) and (20). 


\section{Optimization Problem}

In this section, we determine the Hamilton-Jacobi-Bellman equation for the surplus process. We define the following differential operator:

$$
\mathcal{L}=\frac{1}{2} c^{2} y^{2}\left(\sigma_{Y} \sigma_{Y}^{\prime}+\sigma_{Y_{3}}^{2}\right) \frac{\partial^{2}}{\partial y^{2}}+c \beta y \frac{\partial}{\partial y} .
$$

We define the general value function

$$
G(t, x, y)=E[u(T, X, Y) \mid X(t)=x, Y(t)=y]
$$

where $G(t, x, y)$ is the path of $X(t)$ and $Y(t)$ given the portfolio strategy $\Delta(t)=\left(\Delta_{B}(t), \Delta_{S}(t)\right)$. Let $U(t, x, y)$ be a convex function in $X(t)$ and $Y(t)$ such that

$$
\begin{aligned}
& U(t, x, y)=\min _{\triangle, B, C} E[U(T, X, Y) \mid X(t)=x, Y(t)=y]=\min _{\triangle, B, C} G(t, x, y), \\
& \text { subject to (16). }
\end{aligned}
$$

Then $U(t, x, y)$ satisfies the HJB equation

$$
\begin{aligned}
& U_{t}+\min _{\Delta, B, C}\left\{-B^{2}(t)+e^{-\eta t} C^{2}(t)+(r x+\Delta(t) \lambda-C(t)-(1-r) B(t)) U_{x}\right. \\
& \left.+\frac{1}{2} \Sigma \Delta(t) \Sigma^{\prime} \Delta(t)^{\prime} U_{x x}+c y \Sigma \Delta(t) \sigma_{Y}^{\prime} U_{x y}\right\}+\mathcal{L} U=0,
\end{aligned}
$$

subject to: $U(T, x, y)=x^{2}-2 \varphi x$.

Since $U$ is a convex function in $X(t)$ and $Y(t)$, and $U(t, x, y) \in C^{1,2}(R \times[0, T])$, then (23) is well defined. Then, the minimizer values of the portfolio strategies, MPB and consumption process are given, respectively by

$$
\begin{gathered}
\Delta^{*}(t)^{\prime}=\frac{\left(\Sigma \Sigma^{\prime}\right)^{-1}\left(-\lambda U_{x}-c y \Sigma \sigma_{Y}^{\prime} U_{x y}\right)}{U_{x x}}, \\
B^{*}(t)=\frac{(1-r) U_{x}}{2}, \\
C^{*}(t)=\frac{U_{x} e^{\eta t}}{2} .
\end{gathered}
$$

Substituting (24), (25) and (26) into (23), we have

$$
\begin{aligned}
& U_{t}+\frac{(1-r)^{2} U_{x}^{2}}{4}+r x U_{x}+c y \beta U_{y}-\frac{(M \lambda)^{\prime} \lambda U_{x}^{2}}{2 U_{x x}}-\frac{U_{x}^{2} e^{\eta t}}{2} \\
& -\frac{c y\left(M \Sigma \sigma_{Y}^{\prime}\right)^{\prime} \lambda U_{x} U_{x y}}{U_{x x}}-\frac{1}{2} c^{2} y^{2} \sigma_{Y} \sigma_{Y}^{\prime} \frac{U_{x y}^{2}}{U_{x x}}+\frac{1}{2} c^{2} y^{2}\left(\sigma_{Y} \sigma_{Y}^{\prime}+\sigma_{Y_{3}}^{2}\right) U_{y y}=0,
\end{aligned}
$$

subject to: $U(T, x, y)=x^{2}-2 \varphi x$,

where $M=\left(\Sigma \Sigma^{\prime}\right)^{-1}$. We assume a quadratic solution of the form:

$$
U(t, x, y)=\phi_{0}(t)+x \phi_{x}(t)+y \phi_{y}(t)+x^{2} \phi_{x x}(t)+x y \phi_{x y}(t)+y^{2} \phi_{y y}(t) .
$$

Now, finding the partial derivatives of (28) with respect to $t, x, y, x y, x x, y y$ and then substitute into (24), (25), (26) we have

$$
\Delta^{*}(t)^{\prime}=\frac{-M \lambda \phi_{x}(t)}{2 \phi_{x x}(t)}-M \lambda x-\frac{y M \lambda \phi_{x y}(t)}{2 \phi_{x x}(t)}-\frac{c y \Sigma \sigma_{Y}^{\prime} \phi_{x y}(t)}{2 \phi_{x x}(t)},
$$




$$
\begin{gathered}
B^{*}(t)=\frac{(1-r)\left(\phi_{x}(t)+2 x \phi_{x x}(t)+y \phi_{x y}(t)\right)}{2}, \\
C^{*}(t)=\frac{\left(\phi_{x}(t)+2 x \phi_{x x}(t)+y \phi_{x y}(t)\right) e^{\eta t}}{2} .
\end{gathered}
$$

Again, using the partial derivatives on (27) and extract the coefficients, we obtained the following ordinary differential equations:

$$
\begin{gathered}
\dot{\phi}_{0}(t)=\frac{(M \lambda)^{\prime} \lambda \phi_{x}^{2}(t)}{4 \phi_{x x}(t)}-\frac{(1-r)^{2} \phi_{x}^{2}}{4}+\frac{a \phi_{x}(t) \phi_{x y}(t)}{2 \phi_{x x}(t)}+\frac{1}{2} \phi_{x}^{2}(t) e^{\eta t}, \phi_{0}(T)=0 \\
\dot{\phi}_{x}(t)=a \phi_{x y}(t)+(M \lambda)^{\prime} \lambda \phi_{x}(t)-r \phi_{x}(t)+\left(2 e^{\eta t}-(1-r)^{2}\right) \phi_{x}(t) \phi_{x x}(t), \phi_{x}(T)=-2 \varphi \\
\dot{\phi}_{y}(t)=\frac{(M \lambda)^{\prime} \lambda \phi_{x}(t) \phi_{x y}(t)}{2 \phi_{x x}(t)}-\frac{(1-r)^{2} \phi_{x}(t) \phi_{x y}(t)}{2}+\frac{a \phi_{x y}^{2}(t)}{2 \phi_{x x}(t)} \\
-c \beta \phi_{y}(t)+\phi_{x}(t) \phi_{x y}(t) e^{\eta t}, \phi_{y}(T)=0 \\
\dot{\phi}_{x x}(t)=(M \lambda)^{\prime} \lambda \phi_{x x}(t)-2 r \phi_{x x}(t)+\left(2 e^{\eta t}-(1-r)^{2}\right) \phi_{x x}^{2}(t), \phi_{x x}(T)=1, \\
\dot{\phi}_{x y}(t)=(M \lambda)^{\prime} \lambda \phi_{x y}(t)-r \phi_{x y}(t)+\left(2 e^{\eta t}-(1-r)^{2}\right) \phi_{x x}(t) \phi_{x y}(t)-c \beta \phi_{x y}(t), \phi_{x y}(T)=0, \\
\dot{\phi}_{y y}(t)=\frac{(M \lambda)^{\prime} \lambda \phi_{x y}^{2}(t)}{4 \phi_{x x}(t)}-\frac{(1-r)^{2} \phi_{x y}^{2}}{4}+2 c \beta \phi_{y y}(t) \\
-\frac{1}{2} c^{2} y^{2}\left(\sigma_{Y} \sigma_{Y}^{\prime}+\sigma_{Y_{3}}^{2}\right)\left(2 \phi_{y y}(t)-\frac{\phi_{x y}^{2}(t)}{2 \phi_{x x}(t)}\right)+\frac{1}{2} \phi_{x y}(t)^{2}, \phi_{y y}(T)=0
\end{gathered}
$$

where $a=c\left(M \Sigma \sigma_{Y}^{\prime}\right)^{\prime} \lambda, k=(M \lambda)^{\prime} \lambda$.

Obviously, from (34), we have that $\phi_{x y}(t)=0$. Solving (33), we have

$$
\phi_{x x}(t)=\frac{(k-2 r)(k-2 r+\eta) e^{(2 r-k)(T-t)}}{H(t)}=f(t) .
$$

where $H(t)=2(k-2 r)\left[e^{\eta T}-e^{\eta t}\right]+(k-2 r+\eta)\left[(1-r)^{2} e^{(2 r-k)(T-t)}+\left(k-1-r^{2}\right)\right]$. We assume that $f(t)>$ 0 , for all time, $t$. Solving (32), we have

$$
\phi_{x}(t)=-2 \varphi e^{-(k-r)(T-t)} g(t)
$$

where $g(t)=\exp \left(\int_{t}^{T}\left[(1-r)^{2}-2 e^{\eta s}\right] f(s) d s\right)$.

\section{Proposition 1}

The triple $\left(\Delta^{*}, B^{*}, C^{*}\right)$ representing the optimal portfolio in the risky assets, optimal minimum benefits and optimal consumption rate is given by

$$
\begin{gathered}
\Delta^{*}(t)^{\prime}=M \lambda\left(\frac{\varphi e^{-(k-r)(T-t)} g(t)}{f(t)}-X^{*}(t)\right), \\
B^{*}(t)=(1-r)\left(X^{*}(t) f(t)-\varphi g(t) e^{-(k-r)(T-t)}\right), \\
C^{*}(t)=\left(X^{*}(t) f(t)-\varphi g(t) e^{-(k-r)(T-t)}\right) e^{\eta t} .
\end{gathered}
$$

Proof. Using the value of $\phi_{x}(t), \phi_{x x}(t)$ and $\phi_{x y}(t)$ into (29)-(31), the results follow.

The efficient portfolio strategies (37) depend on the term $\frac{\varphi g(t) e^{-(k-r)(T-t)}}{f(t)}-X^{*}(t)$. Using the definition of $\varphi$, we have the following:

$$
\Delta^{*}(t)^{\prime}=M \lambda\left(\frac{g(t) e^{-(k-r)(T-t)}}{2 \xi f(t)}+\frac{E\left(X^{*}(T)\right) g(t) e^{-(k-r)(T-t)}}{2 \xi f(t)}-X^{*}(t)\right) .
$$

Stat., Optim. Inf. Comput. Vol. 6, December 2018 


$$
\begin{aligned}
B^{*}(t)=(1-r)\left(X^{*}(t) f(t)-\varphi g(t) e^{-(k-r)(T-t)}\right) & \\
C^{*}(t) & =\left(X^{*}(t) f(t)-\frac{g(t) e^{-(k-r)(T-t)}}{2 \xi}-\frac{E\left(X^{*}(T)\right) g(t) e^{-(k-r)(T-t)}}{2 \xi}\right) .
\end{aligned}
$$

These show that the optimal investment strategy, optimal MPB and optimal consumption can be expressed in terms of expected final surplus.

Notice that the portfolio process can be expressed as a function of optimal consumption and also optimal MPB and vice versa as follows:

$$
\begin{gathered}
\Delta^{*}(t)^{\prime}=-\frac{M \lambda}{f(t)} C^{*}(t) e^{-\eta t}=-\frac{M \lambda}{f(t)}(1-r) \tilde{C}^{*}(t) e^{-\eta t}=\frac{M \lambda B^{*}(t)}{f(t)(1-r)} . \\
B^{*}(t)=(1-r) C^{*}(t) e^{-\eta t}=(1-r)^{2} \tilde{C}^{*}(t) e^{-\eta t}=\Delta^{*}(t)(M \lambda)^{-1}(1-r) f(t) .
\end{gathered}
$$

These show that the value of the control variables have significant influence on one another. Observe that a linear relationship exists between investment strategies, $\Delta^{*}$ and consumption plan, $\tilde{C}^{*}$.

\section{First and Second Moments of the Optimal Surplus}

Under the optimal feedback control (37), (38) and (39), the stochastic differential equation for optimal surplus process $X^{*}(t)$ obtained in (16) is

$$
\begin{aligned}
& d X^{*}(t)=\left(\left(r-k-f(t)\left(e^{\eta t}-(1-r)^{2}\right)\right) X^{*}(t)+c \beta Y(t)\right. \\
& \left.+\varphi g(t) e^{-(k-r)(T-t)}\left(\frac{k}{f(t)}+e^{\eta t}+(1-r)^{2}\right)\right) d t \\
& +\left(c \sigma_{Y}^{\prime} Y(t)+\Sigma^{\prime} M \lambda\left(\frac{\varphi g(t) e^{-(k-r)(T-t)}}{f(t)}-X^{*}(t)\right)\right)^{\prime} d W(t)+c \sigma_{Y_{3}} Y(t) d W^{Y}(t), \\
& X^{*}(0)=x_{0} .
\end{aligned}
$$

Applying the Itô formula to $X^{* 2}(t)$, we obtain following

$$
\begin{aligned}
& d X^{* 2}(t)=\left\{\left(2\left(r-f(t)\left(e^{\eta t}-(1-r)^{2}\right)-k\right) X^{* 2}(t)\right.\right. \\
& +\left(2 c \beta-2 c \sigma_{Y} \Sigma^{\prime} M \lambda\right) Y(t) X^{*}(t)+2 \varphi g(t) X^{*}(t) e^{-(k-r)(T-t)}\left(e^{\eta t}+(1-r)^{2}\right) \\
& +2 c \sigma_{Y} \Sigma^{\prime} M \lambda \frac{g(t) Y(t)}{f(t)} e^{-(k-r)(T-t)}+c^{2}\left(\sigma_{Y} \sigma_{Y}^{\prime}+\sigma_{Y_{3}}^{2}\right) Y^{2}(t) \\
& \left.+\frac{k \varphi^{2} g(t)^{2}}{f(t)^{2}} e^{-2(k-r)(T-t)}\right\} d t+2 c \sigma_{Y_{3}} Y(t) X^{*}(t) d W^{Y}(t) \\
& +2\left(c \sigma_{Y}^{\prime} Y(t) X^{*}(t)+\Sigma^{\prime} M \lambda X^{*}(t)\left(\frac{\varphi g(t) e^{-(k-r)(T-t)}}{f(t)}-X^{*}(t)\right)\right)^{\prime} d W(t), \\
& X^{* 2}(0)=x_{0}^{2} .
\end{aligned}
$$

Applying the Ito formula to the product of $X^{*}(t)$ and $Y(t)$, we obtain the following

$$
\begin{aligned}
& d\left(Y(t) X^{*}(t)\right)=\left\{\left(\beta-k+r-f(t)\left(e^{\eta t}-(1-r)^{2}\right)-\sigma_{Y}\left(\Sigma^{\prime} M \lambda\right)^{\prime}\right) Y(t) X^{*}(t)\right. \\
& \left.+\varphi g(t) Y(t) e^{-(k-r)(T-t)}\left(\frac{k+\sigma_{Y}\left(\Sigma^{\prime} M \lambda\right)^{\prime}}{f(t)}+e^{\eta t}+(1-r)^{2}\right)+c\left(\beta+\sigma_{Y} \sigma_{Y}^{\prime}+\sigma_{Y_{3}}^{2}\right) Y^{2}(t)\right\} d t \\
& +\left(\sigma_{Y}^{\prime} Y(t) X^{*}(t)+c \sigma_{Y}^{\prime} Y^{2}(t)+\Sigma^{\prime} M \lambda Y(t)\left(\frac{\varphi g(t) e^{-(k-r)(T-t)}}{f(t)}-X^{*}(t)\right)\right)^{\prime} d W(t) \\
& +\sigma_{Y_{3}} Y(t)(c Y(t)+X(t)) d W^{Y}(t), \\
& Y(t) X^{*}(0)=x_{0} y_{0} .
\end{aligned}
$$


Taking the mathematical expectation of (40), (41) and (42), we obtain the following ordinary differential equations

$$
\begin{aligned}
& d E\left(X^{*}(t)\right)=\left(\left(r-k-f(t)\left(e^{\eta t}-(1-r)^{2}\right)\right) E\left(X^{*}(t)\right)+c \beta E(Y(t))\right. \\
& \left.+\varphi g(t) e^{-(k-r)(T-t)}\left(\frac{k}{f(t)}+e^{\eta t}+(1-r)^{2}\right)\right) d t \\
& E\left(X^{*}(0)\right)=x_{0}, \\
& d E\left(X^{* 2}(t)\right)=\left\{\left(2\left(r-f(t)\left(e^{\eta t}-(1-r)^{2}\right)-k\right) E\left(X^{* 2}(t)\right)\right.\right. \\
& +(2 c \beta-2 c \alpha) E\left(Y(t) X^{*}(t)\right)+2 \varphi g(t) E\left(X^{*}(t)\right) e^{-(k-r)(T-t)}\left(e^{\eta t}+(1-r)^{2}\right) \\
& \left.+2 c \alpha \frac{g(t) E(Y(t))}{f(t)} e^{-(k-r)(T-t)}+c^{2}\left(\sigma_{Y} \sigma_{Y}^{\prime}+\sigma_{Y_{3}}^{2}\right) E\left(Y^{2}(t)\right)+\frac{k \varphi^{2} g(t)^{2}}{f(t)^{2}} e^{-2(k-r)(T-t)}\right\} d t, \\
& E\left(X^{* 2}(0)\right)=x_{0}^{2}, \\
& d E\left(Y(t) X^{*}(t)\right)=\left\{\left(\beta-k+r-f(t)\left(e^{\eta t}-(1-r)^{2}\right)-\alpha\right) E\left(Y(t) X^{*}(t)\right)\right. \\
& \left.+\varphi g(t) E(Y(t)) e^{-(k-r)(T-t)}\left(\frac{k+\alpha}{f(t)}+e^{\eta t}+(1-r)^{2}\right)+c\left(\beta+\sigma_{Y} \sigma_{Y}^{\prime}+\sigma_{Y_{3}}^{2}\right) E\left(Y^{2}(t)\right)\right\} d t, \\
& E\left(Y(0) X^{*}(0)\right)=x_{0} y_{0},
\end{aligned}
$$

where $\alpha=\sigma_{Y}\left(\Sigma^{\prime} M \lambda\right)$. From (6), we have that

$$
E(Y(t))=y_{0} e^{\beta t}, E\left(Y^{2}(t)\right)=y_{0}^{2} e^{\left(2 \beta+\sigma_{Y} \sigma_{Y}^{\prime}+\sigma_{Y_{3}}^{2}\right) t} .
$$

Solving (43), we obtain the first moment, $E\left(X^{*}(t)\right)$ of $X^{*}(t)$ as

$$
E\left(X^{*}(t)\right)=x_{0} e^{(r-k) t-Q(t)}+c \beta y_{0} L_{1}(t) e^{(r-k) t-Q(t)}+\varphi L_{2}(t) e^{(r-k)(T+t)-Q(t)},
$$

where $Q(t)=\int_{0}^{t} f(s)\left(e^{\eta s}-(1-r)^{2}\right) d s, L_{1}(t)=\int_{0}^{t} e^{(\beta-r+k) s+Q(s)} d s$

$L_{2}(t)=\int_{0}^{t} g(s) e^{-2(r-k) s+Q(s)}\left(\frac{k}{f(s)}+e^{\eta s}+(1-r)^{2}\right) d s$.

At time $t=T$, we have

$$
E\left(X^{*}(T)\right)=\tilde{x}_{0} \epsilon+\varphi \nu
$$

where

$$
\begin{gathered}
\epsilon=e^{(r-k) T-Q(T)}, \\
\tilde{x}_{0}=x_{0}+c \beta y_{0} L_{1}(T), \\
\nu=\epsilon L_{2}(T) e^{(r-k) T} .
\end{gathered}
$$

Solving (44), we obtain the following

$$
\begin{aligned}
& E\left(Y(t) X^{*}(t)\right)=x_{0} y_{0} e^{(\beta-k+r-\alpha) t-Q(t)}+\varphi y_{0} N_{1}(t) e^{-(k-r) T} e^{(\beta-k+r-\alpha) t-Q(t)} \\
& +c y_{0}\left(\beta+\sigma_{Y} \sigma_{Y}^{\prime}+\sigma_{Y_{3}}^{2}\right) N_{2}(t) e^{(\beta-k+r-\alpha) t-Q(t)}
\end{aligned}
$$

where $N_{1}(t)=\int_{0}^{t} g(s) e^{-\alpha s+Q(s)}\left(\frac{k+\alpha}{f(s)}+e^{\eta s}+(1-r)^{2}\right) d s$

and $N_{2}(t)=\int_{0}^{t} e^{\left(\beta+k-r+\alpha+\sigma_{Y} \sigma_{Y}^{\prime}+\sigma_{Y_{3}}^{2}\right) s+Q(s)} d s$. Solving (44), we obtain the second moment $E\left(X^{* 2}(t)\right)$ of $X^{* 2}(t)$ as

$$
\begin{aligned}
& E\left(X^{* 2}(t)\right)=x_{0}^{2} e^{(2 r-k) t-2 Q(t)}+M_{1}(t) e^{(2 r-k) t-2 Q(t)}+ \\
& 2 \varphi M_{2}(t) e^{2(r-k) T} e^{(2 r-k) t-2 Q(t)}+\varphi^{2} e^{2(r-k) T} e^{(2 r-k) t-2 Q(t)} M_{3}(t)
\end{aligned}
$$

where

$$
M_{1}(t)=\int_{0}^{t}\left(2 c(\beta-\alpha)\left(x_{0} y_{0}+c y_{0}\left(\beta+\sigma_{Y} \sigma_{Y}^{\prime}+\sigma_{Y_{3}}^{2}\right) N_{2}(s)\right) e^{(\beta-r-\alpha) s+Q(s)}+\right.
$$

$\left.\frac{2 c \alpha y_{0} g(s)}{f(s)} e^{-(k-r)(T-s)} e^{(\beta-2 r+k) s+2 Q(s)}+c^{2} y_{0}^{2}\left(\sigma_{Y} \sigma_{Y}^{\prime}+\sigma_{Y_{3}}^{2}\right) e^{\left(2 \beta-2 r+k+\sigma_{Y} \sigma_{Y}^{\prime}+\sigma_{Y_{3}}^{2}\right) s+2 Q(s)}\right) d s$, 


$$
\begin{aligned}
& M_{2}(t)=\int_{0}^{t}\left(g(s)\left(e^{\eta s}+(1-r)^{2}\right) e^{-(k-r) T}\left(x_{0}+c y_{0} \beta L_{1}(s)\right) e^{-(2 r-k) s+Q(s)}+c y_{0}(\beta-\right. \\
&\left.\alpha) N_{1}(s) e^{-(k-r) T} e^{(\beta-r-\alpha) s+Q(s)}\right) d s, \\
& M_{3}(t)=\int_{0}^{t}\left(2 g(s)\left(e^{\eta s}+(1-r)^{2}\right) L_{2}(s) e^{-(2 r-k) s+Q(s)}+\frac{k^{2} g(s)^{2}}{f(s)^{2}} e^{2(k-r) T} e^{-(2 r-k) s+2 Q(s)}\right) d s .
\end{aligned}
$$

At time $t=T$, we have

$$
E\left(X^{* 2}(T)\right)=\epsilon^{2} M_{3}(T) e^{2(r-k) T}\left[\frac{x_{0}^{2}+M_{1}(T) e^{-2(r-k) T}}{M_{3}(T)}+2 \varphi \frac{M_{2}(T)}{M_{3}(T)}+\varphi^{2}\right] .
$$

\section{The Efficient Frontier}

We now establish the efficient frontier of the process that measure the level of risk given the expected target of the final surplus.

$$
\begin{aligned}
\operatorname{Var}\left(X^{*}(T)\right) & =E\left(X^{* 2}(T)\right)-\left(E\left(X^{*}(T)\right)\right)^{2} \\
& =\epsilon^{2} M_{3}(T) e^{2(r-k) T} \\
& \times\left[\frac{x_{0}^{2}+M_{1}(T) e^{-2(r-k) T}}{M_{3}(T)}+2 \varphi \frac{M_{2}(T)}{M_{3}(T)}+\varphi^{2}\right]-\left(E\left(X^{*}(T)\right)\right)^{2} \\
& =\epsilon^{2} M_{3}(T) e^{2(r-k) T} \\
& \times\left[\frac{x_{0}^{2}+M_{1}(T) e^{-2(r-k) T}}{M_{3}(T)}+2 \varphi \frac{M_{2}(T)}{M_{3}(T)}+\varphi^{2}-\frac{\left(E\left(X^{*}(T)\right)\right)^{2} e^{-2(r-k) T}}{\epsilon^{2} M_{3}(T)}\right] \\
& =\epsilon^{2} M_{3}(T) e^{2(r-k) T}\left[\frac{x_{0}^{2}+M_{1}(T) e^{-2(r-k) T}}{M_{3}(T)}+2 \frac{1}{\nu}\left(E\left(X^{*}(T)\right)-\tilde{x}_{0} \epsilon\right) \frac{M_{2}(T)}{M_{3}(T)}\right. \\
& +\frac{1}{\nu^{2}}\left(E\left(X^{*}(T)\right)-\tilde{x}_{0} \epsilon\right)^{2}-\frac{e^{-2(r-k) T}}{\epsilon^{2} M_{3}(T)}\left\{\left(E\left(X^{*}(T)\right)-\tilde{x}_{0} \epsilon\right)^{2}\right. \\
& \left.\left.+2 \tilde{x}_{0} \epsilon E\left(X^{*}(T)\right)-\tilde{x}_{0}^{2} \epsilon^{2}\right\}\right] \\
& =\frac{\psi_{1}}{\pi}-\frac{\psi_{2}^{2}}{\pi^{2}}+\left(\psi_{2}-\tilde{x}_{0} \pi \epsilon+\pi E\left(X^{*}(T)\right)\right)^{2},
\end{aligned}
$$

where

$$
\begin{gathered}
\psi_{1}=\epsilon^{2} e^{2(r-k) T}\left(x_{0}^{2}+M_{1}(T) e^{-2(r-k) T}\right)+\tilde{x}_{0}^{2} \epsilon^{2}, \\
\psi_{2}=\frac{\epsilon^{2} M_{2}(T) e^{2(r-k) T}}{\nu}+\tilde{x}_{0} \epsilon, \\
\pi=\frac{\epsilon^{2} M_{3}(T) e^{2(r-k) T}}{\nu^{2}}-1 .
\end{gathered}
$$

Let $\sigma_{X^{*}}(T)=\sqrt{\operatorname{Var}\left(X^{*}(T)\right)}$. The expected optimal final surplus and its standard deviation, $\sigma_{X^{*}}(T)$, are related by

$$
E\left(X^{*}(T)\right)=\tilde{x}_{0} \epsilon-\frac{\psi_{2}}{\pi}+\frac{1}{\pi} \sqrt{\sigma_{X}^{2}(T)+\frac{\psi_{2}^{2}}{\pi^{2}}-\frac{\psi_{1}}{\pi}} .
$$

Obviously, the efficient frontier (52) is hyperbolic in shape. Since the efficient frontier may not be linear, we concludes that short-selling is not allowed and borrowing is restricted.

Corollary 1

Suppose $\psi_{1}=2 \psi_{2}$, then

$$
E\left(X^{*}(T)\right)=\tilde{x}_{0} \epsilon-\frac{\psi_{2}(\pi+1)}{\pi^{2}}+\frac{1}{\pi} \sigma_{X}(T) .
$$


This implies that if $\psi_{1}=2 \psi_{2}$, the efficient frontier is a straight line with intercept $\tilde{x}_{0} \epsilon-\frac{\psi_{2}(\pi+1)}{\pi^{2}}$ and gradient $\frac{1}{\pi}$.

Using the fact that $E\left(X^{*}(T)\right)=\tilde{x}_{0} \epsilon+\varphi \nu$, we have the following proposition:

\section{Proposition 2}

The optimal portfolio in the risky assets, optimal minimum benefits and optimal consumption rate may be expressed in terms of expected final surplus, $E\left(X^{*}(T)\right)$, respectively as follows:

$$
\begin{gathered}
\Delta^{*}(t)^{\prime}=M \lambda\left(\frac{\left(E\left(X^{*}(T)\right)-\tilde{x}_{0} \epsilon\right) e^{-(k-r)(T-t)} g(t)}{\nu f(t)}-X^{*}(t)\right), \\
B^{*}(t)=(1-r)\left(X^{*}(t) f(t)-\frac{\left(E\left(X^{*}(T)\right)-\tilde{x}_{0} \epsilon\right)}{\nu} g(t) e^{-(k-r)(T-t)}\right), \\
C^{*}(t)=\left(X^{*}(t) f(t)-\frac{\left(E\left(X^{*}(T)\right)-\tilde{x}_{0} \epsilon\right)}{\nu} g(t) e^{-(k-r)(T-t)}\right) e^{\eta t} .
\end{gathered}
$$

Proof. The result follows by using Proposition 1 and (48).

Remark From Proposition 2, we obtain the following:

1. for $t=0$ :

$$
\begin{gathered}
\Delta^{*}(0)^{\prime}=M \lambda\left(\frac{\left(E\left(X^{*}(T)\right)-\tilde{x}_{0} \epsilon\right) e^{-(k-r) T} g(0)}{\nu f(0)}-x_{0}\right), \\
B^{*}(0)=(1-r)\left(x_{0} f(0)-\frac{\left(E\left(X^{*}(T)\right)-\tilde{x}_{0} \epsilon\right)}{\nu} g(0) e^{-(k-r) T}\right), \\
C^{*}(0)=x_{0} f(0)-\frac{\left(E\left(X^{*}(T)\right)-\tilde{x}_{0} \epsilon\right)}{\nu} g(0) e^{-(k-r) T} .
\end{gathered}
$$

2. for $t=T$ :

$$
\begin{gathered}
B^{*}(T)=(1-r)\left(X^{*}(T)-\frac{\left(E\left(X^{*}(T)\right)-\tilde{x}_{0} \epsilon\right)}{\nu}\right), \\
C^{*}(T)=\left(X^{*}(T)-\frac{\left(E\left(X^{*}(T)\right)-\tilde{x}_{0} \epsilon\right)}{\nu}\right) e^{\eta T} .
\end{gathered}
$$

3. at $t=0$, the optimal minimum benefits and optimal consumption will be equal only if $r=0$.

4. at any time $t$, the optimal minimum benefits and optimal consumption will be equal only if $r=1-e^{\eta t}$.

\section{Aggregate Optimal Final Pension Benefits and Optimal Final Consumption Plan}

In this subsection, we consider the aggregate optimal final pension benefits and optimal final consumption plan of a PPM. Now, at time $t=T$ : From $(61$, )we have that the optimal final MPB is

$$
\begin{aligned}
& B^{*}(T)=(1-r)\left(X^{*}(T)-\frac{E\left(X^{*}(T)\right)}{L_{2}(T)} e^{-2(r-k) T+Q(T)}\right. \\
& \left.+\frac{1}{L_{2}(T)}\left(x_{0}+c y_{0} \beta L_{1}(T)\right) e^{-(r-k) T}\right) .
\end{aligned}
$$

Therefore, the aggregate optimal final pension benefits that will accrue to the PPM is

$$
\begin{aligned}
& F^{*}(T)=B^{*}(T)+(1-h) X^{*}(T)=(r-h) X^{*}(T)- \\
& (1-r)\left[\frac{E\left(X^{*}(T)\right)}{L_{2}(T)} e^{-2(r-k) T+Q(T)}+\frac{1}{L_{2}(T)}\left(x_{0}+c y_{0} \beta L_{1}(T)\right) e^{-(r-k) T}\right] .
\end{aligned}
$$


Interestingly, the aggregate pension benefits depend on the shared part of optimal surplus at time, $T$, the expected value of optimal final surplus that grows as the financial market grows, initial surplus, the contributions of the PPM which are also influence by the market behaviour and consumption rate. Observe that if $h=r$, (62) becomes

$$
F^{*}(T)=(1-r)\left[\frac{E\left(X^{*}(T)\right)}{L_{2}(T)} e^{-2(r-k) T+Q(T)}+\frac{1}{L_{2}(T)}\left(x_{0}+c y_{0} \beta L_{1}(T)\right) e^{-(r-k) T}\right],
$$

which is independent of the optimal surplus at time, $T$ but depends on the expected value of optimal final surplus. From (62), we observe that if $r>h$, the aggregate optimal final pension benefits will increase and decrease if $r<h$.

The optimal terminal consumption process is obtained as

$$
\begin{aligned}
& C^{*}(T)=\left(X^{*}(T)-\frac{E\left(X^{*}(T)\right)}{L_{2}(T)} e^{-2(r-k) T+Q(T)}\right. \\
& \left.+\frac{1}{L_{2}(T)}\left(x_{0}+c y_{0} \beta L_{1}(T)\right) e^{-(r-k) T}\right) e^{\eta T}
\end{aligned}
$$

It is also observed that if

$$
X^{*}(T)>\frac{E\left(X^{*}(T)\right)}{L_{2}(T)} e^{-2(r-k) T+Q(T)}+\frac{1}{L_{2}(T)}\left(x_{0}+c y_{0} \beta L_{1}(T)\right) e^{-(r-k) T}
$$

the optimal final consumption plan will be nonnegative and negative if

$$
X^{*}(T)<\frac{E\left(X^{*}(T)\right)}{L_{2}(T)} e^{-2(r-k) T+Q(T)}+\frac{1}{L_{2}(T)}\left(x_{0}+c y_{0} \beta L_{1}(T)\right) e^{-(r-k) T} .
$$

Again, if

$$
X^{*}(T)=\frac{E\left(X^{*}(T)\right)}{L_{2}(T)} e^{-2(r-k) T+Q(T)}+\frac{1}{L_{2}(T)}\left(x_{0}+c y_{0} \beta L_{1}(T)\right) e^{-(r-k) T} .
$$

it implies that there will be zero consumption.

The following proposition gives the total expected discounted value of optimal consumption and MPB.

\section{Proposition 3}

The total expected discounted value of the optimal consumption plan, optimal MPB and optimal aggregate pension benefits in the time interval $[0, T]$, denoted by $\hat{C}, \hat{B}$ and $\hat{F}$, respectively, are given by

$$
\begin{aligned}
\hat{C} & =E \int_{0}^{T} e^{-r t} C^{*}(t) d t \\
& =\int_{0}^{T} E\left(X^{*}(t)\right) f(t) e^{(\eta-r) t} d t-K \int_{0}^{T} g(t) e^{(k+\eta-2 r) t} d t, \\
\hat{B} & =E \int_{0}^{T} e^{-r t} B^{*}(t) d t \\
& =(1-r)\left[\int_{0}^{T} E\left(X^{*}(t)\right) f(t) e^{-r t} d t-K \int_{0}^{T} g(t) e^{(k-2 r) t} d t\right], \\
\hat{F}= & E \int_{0}^{T} e^{-r t} F^{*}(t) d t \\
= & \int_{0}^{T}[1-h+(1-r) f(t)] E\left(X^{*}(t)\right) e^{-r t} d t-K \int_{0}^{T} g(t) e^{(k-2 r) t} d t,
\end{aligned}
$$

where $K=\frac{\left(E\left(X^{*}(T)\right)-\tilde{x}_{0} \epsilon\right) e^{-(k-r) T}}{\nu}$. 
Proof. The results follow by multiplying (64), (61) by $e^{-r t}$, then integrating with respect to $t$ from 0 to $T$ and setting $T=t$ in (62) and multiple by $e^{-r t}$, then integrating with respect to $t$ from 0 to $T$.

Remark 3

If the PPM retires voluntarily from the scheme, then the final aggregate benefit is

$$
\begin{aligned}
& \hat{F}=\int_{0}^{T_{0}}[1-h+(1-r) f(t)] E\left(X^{*}(t)\right) e^{-r t} d t \\
& -\frac{\left(E\left(X^{*}\left(T_{0}\right)\right)-\tilde{x}_{0} \epsilon\right) e^{-(k-r) T_{0}}}{\nu} \int_{0}^{T_{0}} g(t) e^{(k-2 r) t} d t .
\end{aligned}
$$

Suppose the PPM consume continuously throughout his/her life-time, then we have the total consumption to be

$$
\begin{aligned}
\lim _{T \longrightarrow \infty} \hat{C} & =\lim _{T \longrightarrow \infty}\left[\int_{0}^{T} E\left(X^{*}(t)\right) f(t) e^{(\eta-r) t} d t-K \int_{0}^{T} g(t) e^{(k+\eta-2 r) t} d t\right], \\
& =\int_{0}^{\infty} E\left(X^{*}(t)\right) f(t) e^{(\eta-r) t} d t, \text { provided the limits exists. }
\end{aligned}
$$

\section{Numerical Illustration}

In this section, we present some numerical illustration of our results in the above sections in a specific example. This is to enables us to observe the behaviour of the final standard deviation as against the expected final surplus (see figure 1), the initial investment, initial optimal MPB, optimal consumption and the expected optimal final MPB and optimal final total benefits, with respect to the terminal date, sharing rate, $h$ and the expected final surplus. The values of parameters that we consider in this paper, are the following.

- the risk-free rate of interest is $r=0.02$;

- the contribution rate is $c=0.075$;

- the initial salary is $y_{0}=0.9$;

- growth rate of stochastic salary, $\beta=0.0292$;

- correlation coefficient, $\rho=0.3$;

- the consumption preference rate is $\eta=0.01$;

- $\quad$ volatility vector of stochastic salary, $\bar{\sigma}_{Y}=(0.25,0.3,0.2)$;

- volatility matrix of the two risky investment, $\Sigma:=\left(\begin{array}{cc}0.23 & 0 \\ 0.105 & 0.333879\end{array}\right)$, it implies that a Sharpe ratio $\theta=(0.02,0.203367)^{\prime}$;

- $\quad$ initial surplus, $x_{0}=1$;

- $\quad$ growth rate of stock, $\mu=0.09$;

- for table 1 and table $2, t=0$.

Figure 1 shows the parabolic relationship between the expected surplus and its variance at $T=20$ years. The goal of the fund manager is to maximize the expected surplus $E\left(X^{*}(T)\right)$ and simultaneously reduce the variance.

Considering the values of time horizon, $T=1,2,5,8,10$ and 20 years, and given the expected surplus $m=$ $0,1,2, \ldots, 12$, the total amount of initial investment in the risky assets, that is bond and stock, chosen to maximize surplus to the prescribed levels, is shown in Table 1. The investment in cash account is $\Phi_{0}-\left(\Delta_{B}(0)+\Delta_{S}(0)\right)$, which can be obtained from table 1 . Observe from table 1 that the investment strategies increases as the surplus increases, which is expected. Table 2 show the optimal MPB and optimal consumption at the initial time. In table 2, we observe that MPB increases as surplus increases, which is also expected, but consumption reduces. This implies that financial market growth play a vital role in discouraging consumption and thereby increase PPM's benefits at retirement. Table 3 show the expected terminal MPB and aggregate pension benefits for a PPM. We found from our numerical example in table 3 , that as the value of $h$ increases the aggregate benefits reduces and vice versa. 


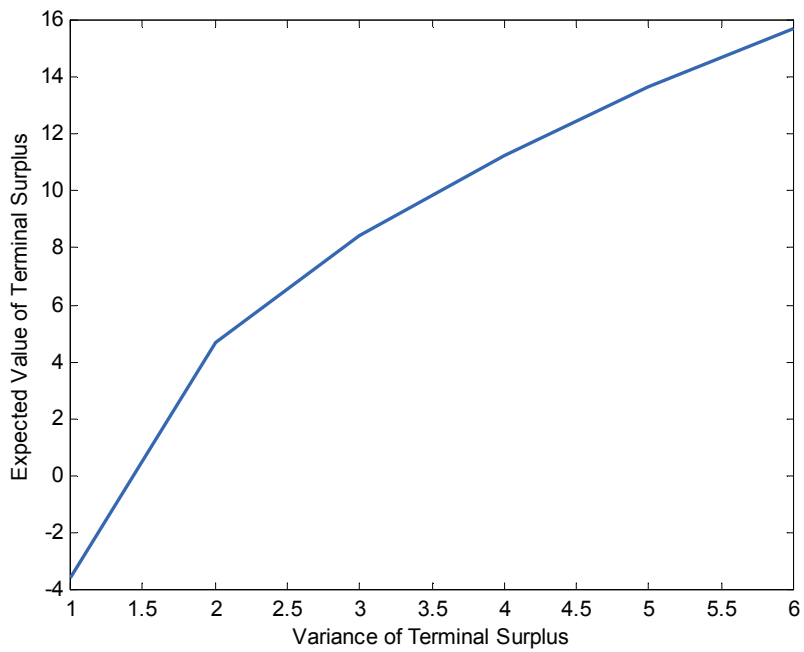

Figure 1. Efficient frontier

Table 1: Initial investment in the risky assets, $\Delta^{* I}(0), \Delta^{* S}(0)$, with $m=E\left(X^{*}(T)\right)$

\begin{tabular}{l|lllllll|lllllll}
\hline \multirow{2}{*}{$m$} & $\Delta^{* I}$ & $\Delta^{* 1}$ & $\Delta^{* 1}$ & $\Delta^{* 1}$ & $\Delta^{* I}$ & $\Delta^{* 1}$ & $\Delta^{* S}$ & $\Delta^{* S}$ & $\Delta^{* S}$ & $\Delta^{* S}$ & $\Delta^{* S}$ & $\Delta^{* S}$ \\
& $T=1$ & $T=2$ & $T=5$ & $T=8$ & $T=10$ & $T=20$ & $T=1$ & $T=2$ & $T=5$ & $T=8$ & $T=10$ & $T=20$ \\
\hline 0 & -0.1927 & -0.1708 & -0.1520 & -0.1415 & -0.1357 & -0.1150 & -1.3912 & -1.2336 & -1.0979 & -1.0217 & -0.9797 & -0.8306 \\
1 & -0.0811 & -0.0780 & -0.0693 & -0.0615 & -0.0570 & -0.0408 & -0.5856 & -0.5632 & -0.5001 & -0.4444 & -0.4114 & -0.8946 \\
2 & 0.0305 & 0.0148 & 0.0135 & 0.0184 & 0.0217 & 0.0334 & 0.2206 & 0.1072 & 0.0976 & 0.1329 & 0.1570 & 0.2413 \\
3 & 0.1422 & 0.1077 & 0.0963 & 0.0984 & 0.1004 & 0.1076 & 1.0268 & 0.7776 & 0.6953 & 0.7103 & 0.7252 & 0.7773 \\
4 & 0.2538 & 0.2005 & 0.1791 & 0.1783 & 0.1791 & 0.1819 & 1.8330 & 1.4480 & 1.2931 & 1.2876 & 1.2935 & 1.3133 \\
5 & 0.3655 & 0.2934 & 0.2618 & 0.2582 & 0.2578 & 0.2561 & 2.6393 & 2.1184 & 1.8908 & 1.8645 & 1.8619 & 1.8492 \\
6 & 0.4771 & 0.3862 & 0.3446 & 0.3382 & 0.3365 & 0.3303 & 3.4455 & 2.7889 & 2.4886 & 2.4422 & 2.4302 & 2.3852 \\
7 & 0.5888 & 0.4790 & 0.4274 & 0.4181 & 0.4152 & 0.4045 & 4.2517 & 3.4592 & 3.0863 & 3.0195 & 2.9985 & 2.9212 \\
8 & 0.7004 & 0.5719 & 0.5102 & 0.4981 & 0.4939 & 0.4787 & 5.0579 & 4.1296 & 3.6840 & 3.5968 & 3.5668 & 3.4571 \\
9 & 0.8121 & 0.6647 & 0.5929 & 0.5780 & 0.5723 & 0.5530 & 5.8642 & 4.8000 & 4.2818 & 4.1741 & 4.1351 & 3.9931 \\
10 & 0.9237 & 0.7575 & 0.6757 & 0.6580 & 0.6513 & 0.6272 & 6.6704 & 5.4705 & 4.8795 & 4.7514 & 4.7034 & 4.5290 \\
11 & 1.0354 & 0.8504 & 0.7585 & 0.7380 & 0.7300 & 0.7014 & 7.4766 & 6.1409 & 5.4773 & 5.3287 & 5.2717 & 5.0650 \\
12 & 1.1470 & 0.9432 & 0.8413 & 0.8179 & 0.8087 & 0.7756 & 8.2828 & 6.8113 & 6.0750 & 5.9061 & 5.8400 & 5.6010 \\
\hline
\end{tabular}

Table 2: Initial MPB, $B^{*}(0)$ and Initial Consumption, $C^{*}(0)$, with $m=E\left(X^{*}(T)\right)$

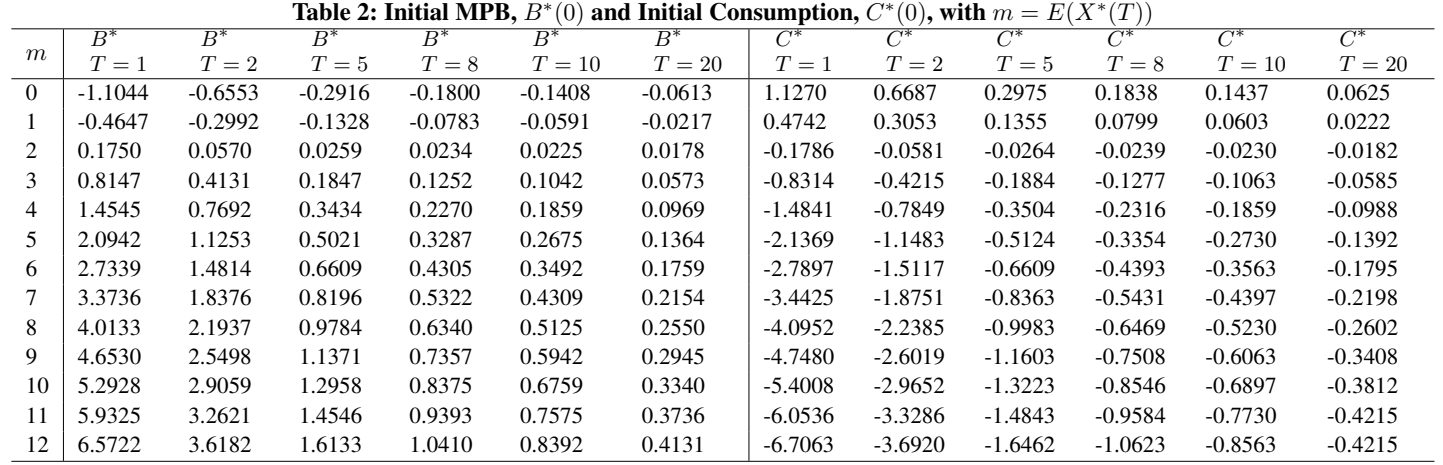


Table 3: Expected Terminal Minimum and Aggregate Pension Benefit, with $m=E\left(X^{*}(T)\right)$

\begin{tabular}{|c|c|c|c|c|c|c|c|c|c|c|c|c|}
\hline$m$ & $\begin{array}{l}h=0.1 \\
B^{*}(T)\end{array}$ & $F^{*}(T)$ & $\begin{array}{l}h=0.2 \\
B^{*}(T)\end{array}$ & $F^{*}(T)$ & $\begin{array}{l}h=0.5 \\
B^{*}(T)\end{array}$ & $F^{*}(T)$ & $\begin{array}{l}h=0.7 \\
B^{*}(T)\end{array}$ & $F^{*}(T)$ & $\begin{array}{l}h=0.8 \\
B^{*}(T)\end{array}$ & $F^{*}(T)$ & $\begin{array}{l}h=0.9 \\
B^{*}(T)\end{array}$ & $F^{*}(T)$ \\
\hline 0 & 0.0013 & 0.0013 & 0.0013 & 0.0013 & 0.0013 & 0.0013 & 0.0013 & 0.0013 & 0.0013 & 0.0013 & 0.0013 & 0.0013 \\
\hline 1 & 0.9779 & 1.8779 & 0.9779 & 1.7779 & 0.9779 & 1.4779 & 0.9779 & 1.2779 & 0.9779 & 1.1779 & 0.9779 & 1.0779 \\
\hline 2 & 1.9546 & 3.7546 & 1.9546 & 3.5546 & 1.9546 & 2.9546 & 1.9546 & 2.5546 & 1.9546 & 2.3546 & 1.9546 & 2.1546 \\
\hline 3 & 2.9313 & 5.6313 & 2.9313 & 5.3313 & 2.9313 & 4.4313 & 2.9313 & 3.8313 & 2.9313 & 3.5313 & 2.9313 & 3.2313 \\
\hline 4 & 3.9080 & 7.5080 & 3.9080 & 7.1080 & 3.9080 & 5.9080 & 3.9080 & 5.1080 & 3.9080 & 4.7080 & 3.9080 & 4.3080 \\
\hline 5 & 4.8847 & 9.3847 & 4.8847 & 8.8847 & 4.8847 & 7.3847 & 4.8847 & 6.3847 & 4.8847 & 5.8847 & 4.8847 & 5.3847 \\
\hline 6 & 5.8614 & 11.2614 & 5.8614 & 10.6614 & 5.8614 & 8.8614 & 5.8614 & 7.6614 & 5.8614 & 7.0614 & 5.8614 & 6.4614 \\
\hline 7 & 6.8380 & 13.1380 & 6.8380 & 12.4380 & 6.8380 & 10.3380 & 6.8380 & 8.9380 & 6.8380 & 8.2380 & 6.8380 & 7.5380 \\
\hline 8 & 7.8147 & 15.0147 & 7.8147 & 14.2147 & 7.8147 & 11.8147 & 7.8147 & 10.2147 & 7.8147 & 9.4147 & 7.8147 & 8.6147 \\
\hline 9 & 8.7914 & 16.8914 & 8.7914 & 15.9914 & 8.7914 & 13.2914 & 8.7914 & 11.4914 & 8.7914 & 10.5914 & 8.7914 & 9.6914 \\
\hline 10 & 9.7681 & 18.7681 & 9.7681 & 17.7681 & 9.7681 & 14.7681 & 9.7681 & 12.7681 & 9.7681 & 11.7681 & 9.7681 & 10.7681 \\
\hline 11 & 10.7448 & 20.6448 & 10.7448 & 19.5448 & 10.7448 & 16.2448 & 10.7448 & 14.0448 & 10.7448 & 12.9448 & 10.7448 & 11.8448 \\
\hline 12 & 11.7214 & 22.5214 & 11.7214 & 21.3214 & 11.7214 & 17.7214 & 11.7214 & 15.3214 & 11.7214 & 14.1214 & 11.7214 & 12.9214 \\
\hline
\end{tabular}

\section{Conclusion}

This paper analyzed the stochastic funding of a DC pension plan that involves minimum pension benefits and aggregate benefits. The objectives are to determine the minimum pension benefits, consumption level and investment strategies that maximize the expected final surplus and at the same time minimizing both the consumption and the variance of the surplus. The problem is formulated as a modified mean-variance optimization problem and solved by means of dynamic programming approach.

The efficient frontier is not linear but has a hyperbolic shape. The optimal investment strategies is made up of three terms. The first term is always nonnegative, increasing with time, and depends inversely on $\xi$, which is the parameter that weighed the relative importance of the fund manager objective of variance minimization with respect to the objective of surplus enhancement. The second and third terms are, respectively the expected value of surplus maximization strategy and optimal surplus that is valued at time $t$. Similarly, optimal MPB and optimal consumption plan depend on three terms. The first term is the optimal surplus which is valued at time, $t$, multiplied by $f(t)$, see (35 for the definition of the functional). The second term is a nonnegative part of the processes that increases with and depend inversely on $\xi$. The third term is the expected value of surplus maximization strategy, multiplied by $g(t)$, see (37 for the definition of the function, $g(t)$ ). Interestingly, show that with the presence of MPB, there exists a linear relationship between the optimal MPB, optimal consumption plan and the vector of optimal investment strategies. We also found that the portfolio process can be expressed as a function of optimal consumption and optimal MPB and vice versa. We further found that the optimal portfolio in the risky assets, optimal minimum benefits and optimal consumption can be express in terms of expected final surplus. The aggregate optimal final pension benefits that will accrued to the PPM was obtained. The aggregate pension benefits was found to depend on the shared part of optimal surplus valued at time, $T$, the expected value of optimal final surplus that grows as the financial market grows, initial surplus, the contributions of the PPM, which are influence by the market behaviour and consumption rate.

The total expected discounted value of optimal consumption and optimal discounted MPB are obtained in proposition 3. PPM life-time consumption process was determined. We found in this paper that short-selling is not allow and borrowing is restricted. A numerical illustration that shows the analytical results proved in this paper were obtained.

\section{REFERENCES}

1. I. Bajeux-Besnainou and R. Portait, Dynamic asset allocation in a mean-variance framework, Management Science, 44, S79-S95, 1998.

2. P. Battocchio and F. Menoncin, Optimal pension management in a stochastic framework, Insurance: Mathematics and Economics, 34, 79-95, 2004.

3. T. Bielecky, H. Jim, S. Pliska and X. Zhou, Continuous-time mean-variance portfolio selection with bankruptcy prohibition, Mathematical Finance, 15, 213-244, 2005.

4. D. Blake, D. Wright and Y. Zhang, Optimal funding and investment strategies in defined contribution pension plans under EpsteinZin utility, Discussion paper, the pensions Institute, Cass Business School, City University, UK, 2008. 
5. J. F. Boulier, S. J. Huang and G. Taillard, Optimal management under stochastic interest rates: the case of a protected defined contribution pension fund, Insurance: Mathematics and Economics, 28, 173-189, 2001.

6. A. J. G. Cairns, D. Blake and K. Dowd, Stochastic lifestyling: Optimal dynamic asset allocation for defined contribution pension plans, Journal of Economic Dynamic and Control, 30, 843-377, 2006.

7. M. Chiu and $\mathrm{D}$. Li, Asset and liability management under a continuous-time mean-variance optimization framework, Insurance: Mathematics and Economics, 39, 330-355, 2006

8. M. Chiu and D. Li, Optimal contributions in a defined benefit pension scheme with stochastic new entrants, Insurance: Mathematics and Economics 37, 335-354, 2005.

9. N. O. Da Cunha and E. Polak, Constrained minimization under vector-valued criteria in finite dimensional spaces, Journal of Mathematical Analysis and Applications, 19, 103-124, 1967.

10. G. Deelstra, M. Grasselli and P. Koehl, Optimal investment strategies in a CIR framework, Journal of Applied Probability, 37, 936-946, 2000

11. P. Devolder, M. Bosch Princep and I. D. Fabian, Stochastic optimal control of annuity contracts, Insurance: Mathematics and Economics, 33, 227-238, 2003.

12. M. Di Giacinto, S. Federico and F. Gozzi, Pension funds with a minimum guarantee: a stochastic control approach, Finance and Stochastic, 2010.

13. J. Gao, Stochastic optimal control of DC pension funds, Insurance: Mathematics and Economics, 42, pp. 1159-1164, 2008.

14. R. Gerrard, S. Haberman and E. Vigna, Optimal investment choices post retirement in a defined contribution pension scheme, Insurance: Mathematics and Economics, 35, 321-342, 2004.

15. S. Haberman and J. H. Sung, Dynamics approaches to pension funding, Insurance: Mathematics and Economics, 15, 151-162, 1994.

16. S. Haberman, Z. Butt and C. Megaloudi, Contribution and solvency risk in a defined benefit pension scheme, Insurance: Mathematics and Economics, 27, 237-259, 2000.

17. S. Haberman and E. Vigna, Optimal investment strategies and risk measures in defined contribution pension schemes, Insurance: Mathematics and Economics, 31, 35-69, 2002.

18. B. H $\phi$ jgaard and E. Vigna, Mean-variance portfolio selection and efficient frontier for defined contribution pension schemes, Technical report R-2007-13, Department of Mathematical Sciences, Aalborg University, 2007.

19. H. C. Huang and A. J. G. Cairns, On the control of defined-benefit pension plans, Insurance: Mathematics and Economics, 38, $113-131,2005$.

20. B. A. Jensen and C. S $\phi$ rensen, Paying for minimum interest guarantees. Who should compensate who?, European Financial Management, 7, 183-211, 1999.

21. R. Josa-Fombellida and J. P. Rincon-Zapatero, Minimization of risks in pension funding by means of contribution and portfolio selection, Insurance: Mathematics and Economics, 29, 35-45, 2001.

22. R. Josa-Fombellida and J. P. Rincon-Zapatero, Optimal risk management in defined benefit stochastic pension funds, Insurance: Mathematics and Economics, 34, 489-503, 2004.

23. R. Josa-Fombellida and J. P. Rincon-Zapatero, Mean-variance portfolio and contribution selection in stochastic pension funding European Journal of Operational Research, 187, 120-137, 2008.

24. R. Korn and M. Krekel, Optimal portfolios with fixed consumption or income streams, Working paper, University of Kaiserslautern, 2001.

25. D. Li and W. Ng, Optimal dynamic portfolio selection: multiperiod mean-variance formulation, Mathematical Finance, 10, 387-406, 2000 .

26. H. Markowitz, Portfolio selection, Journal of finance, 7, 77-91, 1952.

27. H. Markowitz, Portfolio selection: efficient diversification of investments, New York, Wiley, 1959.

28. R. C. Merton, Optimal consumption and portfolio rules in a continuous-time model, Journal of Economic Theory, 3, 373-413, 1971.

29. C. I. Nkeki, Mean-variance portfolio selection with inflation hedging strategy: a case of a defined contributory pension scheme, Theory and Applications of Mathematics \& Computer Science, 2(2), 67-82, 2012.

30. C. I. Nkeki, Mean-variance portfolio selection problem with time-dependent salary for defined contribution pension scheme, Financial Mathematics and Applications, 3(1), 1-26, 2013a.

31. C. I. Nkeki and C. R. Nwozo, Mean-variance portfolio optimization problem with fixed salary and inflation protection for a defined contribution pension scheme, Journal of Statistical and Econometric Methods, 2(2), 157-173, 2013.

32. C. I. Nkeki, Mean-variance portfolio selection problem with stochastic salary for a defined contribution pension scheme: a stochastic linear-quadratic framework, Statistics Opt. \& Inform. Comput., 1, 62-81, $2013 \mathrm{~b}$.

33. C. I. Nkeki, Stochastic funding of a defined contribution pension plan with proportional administrative costs and taxation under mean-variance optimization approach, Statistics Opt. \& Inform. Comput., 2, 323-338, 2014.

34. C. I. Nkeki, Optimal investment and optimal additional voluntary contribution rate of a DC pension fund in a jump-diffusion environment, Annals of Financial Economics, 2017. DOI: 10.1142/S2010495217500178.

35. C. I. Nkeki, Optimal pension fund management in a jumpdiffusion environment: Theoretical and empirical studies, Journal of Computational and Applied Mathematics, 330, 228-252, 2018.

36. H. Richardson, A minimum variance result in continuous trading portfolio optimization, Management Science, 35, 1045-1055, 1989.

37. E. Vigna, On efficiency of mean-variance based portfolio selection in DC pension schemes, Collegio Carlo Alberto Notebook, 154, 2010.

38. X. Zhou and D. Li, Continuous-time mean-variance portfolio selection: A stochastic LQ framework, Applied Mathematics and Optimization, 42, 19-33, 2000.

39. A. Zhang, R. Korn and C. O. Ewald, Optimal management and inflation protection for defined contribution pension plans, Working paper, University of St. Andrews, 2007. 